\title{
Overcoming Drug Interference in Transfusion Testing: A Spotlight on Daratumumab
}

\author{
Marilyn T Nedumcheril ${ }^{1,2}$ \\ Robert A DeSimone (D) \\ Sabrina E Racine-Brzostek' \\ Ok Kyong Chaekal ${ }^{1,3}$ \\ Ljiljana V Vasovic (iD) \\ 'Department of Pathology and \\ Laboratory Medicine, New York- \\ Presbyterian Hospital, Weill Cornell \\ Medicine, New York, NY, USA; \\ ${ }^{2}$ New York Blood Center Enterprises, \\ New York, NY, USA; ${ }^{3}$ Department of \\ Medicine/Division of Hematology- \\ Oncology New York-Presbyterian \\ Hospital, Weill Cornell Medicine, \\ New York, NY, USA
}

\begin{abstract}
Daratumumab, a monoclonal antibody therapeutic, is highly efficacious and widely used in all stages of multiple myeloma and amyloidosis and has promising activity in other hematologic disorders. Daratumumab interacts with red blood cells, interfering with pre-transfusion testing. This interference can lead to compromising transfusion safety, extensive blood bank work ups and delays in provision of compatible units. Several methods have been developed to negate daratumumab interference with indirect antiglobulin testing. They are based on i) standard blood bank techniques including dithiothreitol and enzymatic treatment of reagent cells, using reagent red blood cells negative for CD38, ii) blocking CD38 antigens on reagent or donor cells, iii) neutralization of anti-CD38 antibody in patient plasma prior to testing, and iv) extended antigen typing of patient red blood cells in conjunction with provision of phenotypically matched units for transfusion. Implementation of those methods by the blood bank should be a planned effort coordinated with the patient's clinical team. Timely involvement of blood bank and transfusion services and educational efforts by both blood banks and clinical providers can improve the overall daratumumab safety profile in regard to blood transfusion.
\end{abstract}

Keywords: immunohematology, CD38, drug neutralization, dithiothreitol, incompatible crossmatch, indirect antiglobulin testing

\section{Introduction}

Pre-transfusion testing is a cornerstone of safe blood transfusion practices. ${ }^{1}$ Principal components of pre-transfusion testing consist of recipient's and donor's blood typing, detection of red blood cell (RBC) antibodies in recipient's plasma, selection of compatible blood units including extended typing beyond $\mathrm{ABO} / \mathrm{Rh}$ when clinically significant alloantibodies are identified, and finally confirming compatibility by serologic crossmatching. Interference with pre-transfusion testing can lead to compromised transfusion safety, extensive blood bank work-ups and delays in the provision of compatible RBC units. Drug interference can be observed as a drug-induced immune hemolytic anemia, in either a drug-dependent fashion or through drug-independent antibodies that are serologically indistinguishable from idiopathic warm autoantibodies that can persist after the offending agent is removed or metabolized. A new challenge is presented by a new generation of monoclonal antibody therapeutics such as anti-cluster differentiation (CD)-38 and Anti-CD47 that target antigens expressed by hematological malignancies that are also expressed by RBCs. ${ }^{2,3}$ Most notable is the Anti-CD38 monoclonal antibody daratumumab, targeting multiple myeloma, which has gained wide attention for its clinical utility and will be further discussed. ${ }^{4,5}$
Correspondence: Ljiljana $\vee$ Vasovic Department of Pathology and Laboratory Medicine, New York-Presbyterian Hospital, Weill Cornell Medicine, 525 East 68th Street, Box 25I, New York, NY, 10065, USA

Tel +12127466106

$\mathrm{Fax}+\mathrm{I} 2127468435$

Email ljv9004@med.cornell.edu 


\section{Daratumumab in the Treatment of Plasma Cell Neoplasms}

Plasma cell neoplasms, mostly myeloma but also closely related disorders such as amyloidosis, account for approximately $10 \%$ of all hematologic malignancies and remain largely incurable. In 2021, the American Cancer Society estimates 34,920 newly diagnosed cases and about 12,410 deaths attributed to MM. ${ }^{6}$ Daratumumab is a humanized IgG1 $\kappa$ monoclonal antibody targeting CD38, a surface protein highly expressed in multiple myeloma (MM) cells. Human CD38 antigen is a $46 \mathrm{kDa}$, type II transmembrane glycoprotein which functions as an ADP-ribosyl cyclase. In addition to its expression on plasma cells and malignant myeloma cells, CD38 is also expressed at low levels on other hematopoietic cells, including RBCs and epithelial cells. ${ }^{7}$ Its expression may be stimulated by proinflammatory cytokines in patients with cancer. ${ }^{8}$

Daratumumab effectively induces antibody dependent cellular toxicity (ADCC), complement dependent cytotoxicity, antibody dependent cellular phagocytosis (ADCP), and apoptosis in $\mathrm{MM}$ cells ${ }^{9}$ and has become one of the most effective drugs for treatment of myeloma. ${ }^{10}$ It was first approved by the United States Food and Drug Administration (FDA) for use in adult patients in late 2015. Initially approved as monotherapy for multiple myeloma patients with double-refractory disease who failed at least three prior lines of therapy, it has since been incorporated in front-line treatment and is mostly used in combination with other agents such as proteasome inhibitors, imids and corticosteroids. ${ }^{11,12}$ Such triplet or quadruplet therapies have dramatically improved response, response duration, and life-expectancy of patients with multiple myeloma. Daratumumab is also one of the most active agents approved for treatment of amyloidosis. It has occasionally been used for treatment of delayed RBC engraftment occurring after stem cell transplant, and it has shown promise in treatment of T-ALL. ${ }^{13,14}$ Initially daratumumab was given intravenously. It frequently causes infusionrelated toxicity such as fever, rigors, flushing, and in extreme cases anaphylactic shock. This is prevented by premedication with steroids, antihistamines, and acetaminophen. A more recent formulation combines daratumumab with hyaluronidase (Darzalex Faspro $^{\mathrm{TM}}$, Janssen Biotech, Horsham, PA) and is administered subcutaneously with better tolerance. Premedication is still required. The half-life of daratumumab, like that of other antibody treatments, is measured in weeks. It is dosed weekly or every other week during initial treatment. In many patients this is followed by monthly maintenance treatments so that detectable blood levels are present throughout treatment. Several other CD38 antibody therapies are in development, including isatuximab and MOR202. ${ }^{15}$

No significant clinically detectable hemolysis has been observed to preclude daratumumab therapy in MM patients. However, therapy-related anemia may be observed requiring $\mathrm{RBC}$ transfusion. ${ }^{16,17}$ Interestingly, Schuetz et al. found that daratumumab may be an effective therapeutic agent against autoimmune hemolytic anemia (AIHA) post-hematopoietic stem cell transplantation (HSCT) refractory to established first and second-line therapies. The mechanism of action to induce rapid remission of AIHA post-HSCT is now known, and use should be confined to those with life-threatening disease unresponsive to established therapies. ${ }^{18}$

Daratumumab interference with serological testing in transfusion medicine and other laboratory tests including serum protein and immunofixation electrophoresis and flow cytometry have been readily observed. Despite promising benefits, concerns have been raised regarding daratumumab interference with serological testing and transfusion safety, particularly in urgent situations.

\section{Daratumumab Interferes with Serological Testing in Transfusion Medicine}

Daratumumab does not interfere with $\mathrm{ABO} / \mathrm{RhD}$ typing or with immediate-spin crossmatches. Daratumumab is known to cause interference with serological testing, including red cell antibody screening, antibody identification and cross-matching based on indirect antiglobulin testing (IATs). ${ }^{19}$ Routine pre-transfusion testing algorithm consists of $\mathrm{ABO} / \mathrm{Rh}$ typing, antibody detection and identification, direct antiglobulin test (DAT), and crossmatching whether a patient is on daratumumab or not. ABO typing consists of a forward type that identifies antigens present on the RBC surface by mixing patient's RBCs and antiserum (anti-A and anti-B) and a reverse type to identify what antibodies are present in a patient's plasma/serum by mixing patient plasma with reagent RBCs (A and $\mathrm{B}$ ). $\mathrm{Rh}$ typing is a forward type only. Antibody screens detect the presence of alloantibodies in a patient's plasma by mixing patient plasma with group $\mathrm{O}$ red cells of known phenotype and observing for agglutination. The DAT identifies 
antibodies attached to red blood cells. The patient's red cells are screened by mixed with polyspecific DAT reagent and confirmed by mixing with monospecific anti-IgG and anti-C $3 \mathrm{~b} / \mathrm{d}$ reagents. When the DAT is positive for agglutination with anti-IgG reagent. Elutions may be performed to identify the antibody of interest. Prior to issue of a RBC unit, $\mathrm{RBC}$ crossmatching is performed to check for blood compatibility. When no RBC antibody is detected, pretransfusion testing involves either an electronic crossmatch (computer matches compatible units) or immediate spin to confirm ABO compatibility. Immediate spin crossmatching mixes patient plasma with RBCs from a selected unit at room temperature. However, when an antibody has been detected, an antiglobulin (full or Coomb's) crossmatch is performed by incubating patient plasma, donor RBCs, and AHG reagent at $37 \mathrm{C}$. CD38 expression on reagent red cells will be recognized by daratumumab and will result in a panreactivity in vitro. Initially, it was often confused with an antibody to a high prevalence antigen. Ficintreated red cells, dilution and adsorption techniques failed to eliminate the panreactivity. The direct antiglobulin test (DAT) can be positive following daratumumab therapy. However, studies of patients on daratumumab, where antibody screening yielded positive reactions by IATs, had negative DATs. ${ }^{20}$ Acid eluates failed to show agglutination with patients' own RBCs as well as donor RBCs. These results were observed for two to six months after the last daratumumab infusion. The positivity in IAT testing was not unique to daratumumab but was also seen with other anti-CD38 clones.

In patients taking daratumumab, the DAT is frequently negative and reactivity with the patient's own RBCs is inconsistent, and the auto-control is frequently negative. There is some evidence to suggest that daratumumab induces loss of CD38 on RBCs over time. ${ }^{16}$ This confusion led to unnecessary testing and significant delays in blood provision. In the package insert, the manufacturer recommends that patients should be typed and screened prior to administration of daratumumab. However, another salient point is that blood banks should be consistently notified by providers or pharmacy when a patient is planned to or has received daratumumab. This is also important if daratumumab is used in outpatient setting and for off label use in other lymphoid malignancies. ${ }^{21,22}$ Unrecognized daratumumab interference with blood bank serologic testing can lead not only to unnecessary delays in pre-transfusion testing but also in issuing crossmatch incompatible $\mathrm{RBC}$ units as an emergency release procedure. This raises concerns regarding transfusion safety due to interference with alloantibody identification that can result in an acute or delayed hemolytic transfusion reaction, particularly in urgent situations. Thus, methods to mitigate daratumumab interference were widely investigated and implemented in clinical practice (see Table 1 and Figure 1). Clinicians taking care of patients receiving daratumumab should contact the blood bank to inform them of daratumumab use when ordering a predaratumumab type and screen. Algorithm for clinicians managing patients treated with daratumumab is provided (see Figure 2.)

\section{Methodologies to Negate}

\section{Daratumumab Interference During Pre-Transfusion Testing RBCs with Destroyed or Lacking CD38 Antigens Used for Pre-Transfusion Testing} Prior studies investigating the structure and function of CD38 revealed that the reducing agent dithiothreitol (DTT) can denature CD38, and enzymatic digestion with trypsin can cleave $\mathrm{CD} 38$ from the cell surface. ${ }^{23}$ Interestingly, it has also been reported that samples from patients on anti-CD38 therapy can cause false positivity on subsequent samples via carryover on solid phase instruments such as the TANGO optimo (Bio-Rad Laboratories, Hercules, CA). ${ }^{24}$

To resolve the issue of daratumumab interfering with serological testing, Chapuy et al. investigated methods to prevent binding of daratumumab to $\mathrm{CD} 38$ on reagent RBCs. ${ }^{25}$ As a model they used HL60 cells transduced with either CD38 or green fluorescent protein (CD38 negative control). When exposed to daratumumab spiked plasma, there was a dose dependent binding to CD38+ HL60 cells but not CD38- and controls. When CD38 +HL60 cells are incubated with $10 \mathrm{mmol} / \mathrm{L}$ DTT and $2 \%$ trypsin, daratumumab binding decreases by $92 \%$ and $40 \%$ respectively.

The same researchers compared the eluate reactivity of untreated and DTT-treated RBCs incubated with daratumumab. The eluates of untreated RBCs contained specific binding to CD38+HL60 cells and a mini-5 RBC panel. Eluates of DTT-treated RBCs had no detectable binding to CD38+HL60 cells or the mini-panel of 5 RBCs. DTTsensitive blood groups include Kell, Cartwright, Dombrock, Indian, John Milton Hagen, Knops, Landsteiner-Weiner, Lutheran, and Raph. ${ }^{26}$ However, 
Table I Key Features of Methodologies Utilized to Abrogate Daratumumab Interference in Pretransfusion Testing

I. RBCs with lacking, diminished or destroyed CD38 antigens
used in pre-transfusion testing
Long standing technique
Highly reliable as a blood bank procedure
Training and competency of technologists well established
- Dithiothreitol (DTT)
Denatures CD38 antigen
DTT treated donor cells can be used for a crossmatch
Caution needed as DTT denatures Kell and other clinically
significant antigens
- Trypsin/Papain
Cleaves CD38 antigen
Destroys many other significant RBC antigens
Less reliable than DTT
Cord blood cells
Lacking CD38 antigen
Cord blood cell antigen panels reagent are not routinely
available
- In (Lu) RBCs
Lacking CD38 antigen
Rare and not routinely available reagent cells
Panels from RBC of Daratumumab treated Patients
Not standardized

II. Blocking CD38 antigens on reagent and donor cells

Not recognized by antihuman globulin (AHG)

- Anti-CD38 F(ab')2 fragments

Bind and mask CD38 on reagent cells

Not commercially available.

- Anti-CD38 + monospecific anti-human IgG Pre-Adsorbed RBCs with Daratumumab blocked with monospecific anti-human IgG competing out AHG

- Non-human anti-CD38

Not recognized by AHG

III. Neutralization of anti-CD38 antibody in patient plasma prior to IAT testing

Routine blood bank testing providing adequate anti-CD38 antibody neutralization with:

- Anti-idiotype antibody Commercially available

Not typically available in standard blood bank inventory

- Soluble CD38 antigen

Requires large amounts of soluble CD38 required for neutralization of therapeutic daratumumab levels

Less efficacious than anti-idiotype

Method not standardized

- CD38 antigen from myeloma lines lysate/stroma Difficult to standardize methodology

- Anti-CD38 aptamers
Table I (Continued)

IV. Extended patient antigen typing with phenotypical matching of RBC units

Delays in transfusion if extended-match units difficult to acquire

Historically a method of choice for patients with known auto- and

allo-antibodies

Antibody identification testing not required when matched units

available

Serologic Crossmatch will be incompatible due to presence of AntiCD38

- Extended RBC phenotype of recipients

To be performed prior initiating daratumumab therapy

- RBC genotyping

Can be performed at any time

Universal genotyping is expensive

Extended matching not necessary if:

negative antibody screen and no prior history of auto- and alloantibodies

Abbreviations: AHG, antihuman globulin; DTT, dithiothreitol; IAT, indirect antiglobulin test; RBC, red blood cells.

aside from Kell, the majority of clinically significant alloantibodies can be ruled out. Thus, Kell negative units are administered unless that patient is known to be $\mathrm{K}$ positive. $\mathrm{ABO}$ and $\mathrm{Rh}$ typing are not affected.

Chapuy et al. published a 0.2 M DTT validation study that investigated the applicability of the DTT method to negate daratumumab interference through the Biomedical Excellence for Safer Transfusion (BEST) Collaborative. ${ }^{27}$ The study included 25 worldwide blood bank laboratories in North America, South America, Europe, Australia and the Asia Pacific region. The objective was to determine if the DTT method could reliably permit alloantibody identification in the presence of daratumumab. Regardless of the method of testing, all sites observed daratumumab interference using patients sample with the initial antibody screen with untreated cells. All sites correctly identified an unknown alloantibody in samples spiked with daratumumab. In a follow up survey, $84 \%$ of the respondents agreed that the DTT method fulfills the standard of care when evaluating patients on daratumumab and $89 \%$ of participants said they plan to use the DTT method to test samples of patients on daratumumab.

In order to mitigate destructive effects of $0.2 \mathrm{M}$ DTT on the red cell membrane, particularly the destruction of the Kell antigen, Hosokawa et al. created the Osaka method. ${ }^{28}$ Their method uses $0.01 \mathrm{M}$ DTT in $\mathrm{pH} 7.3$

(Continued) 


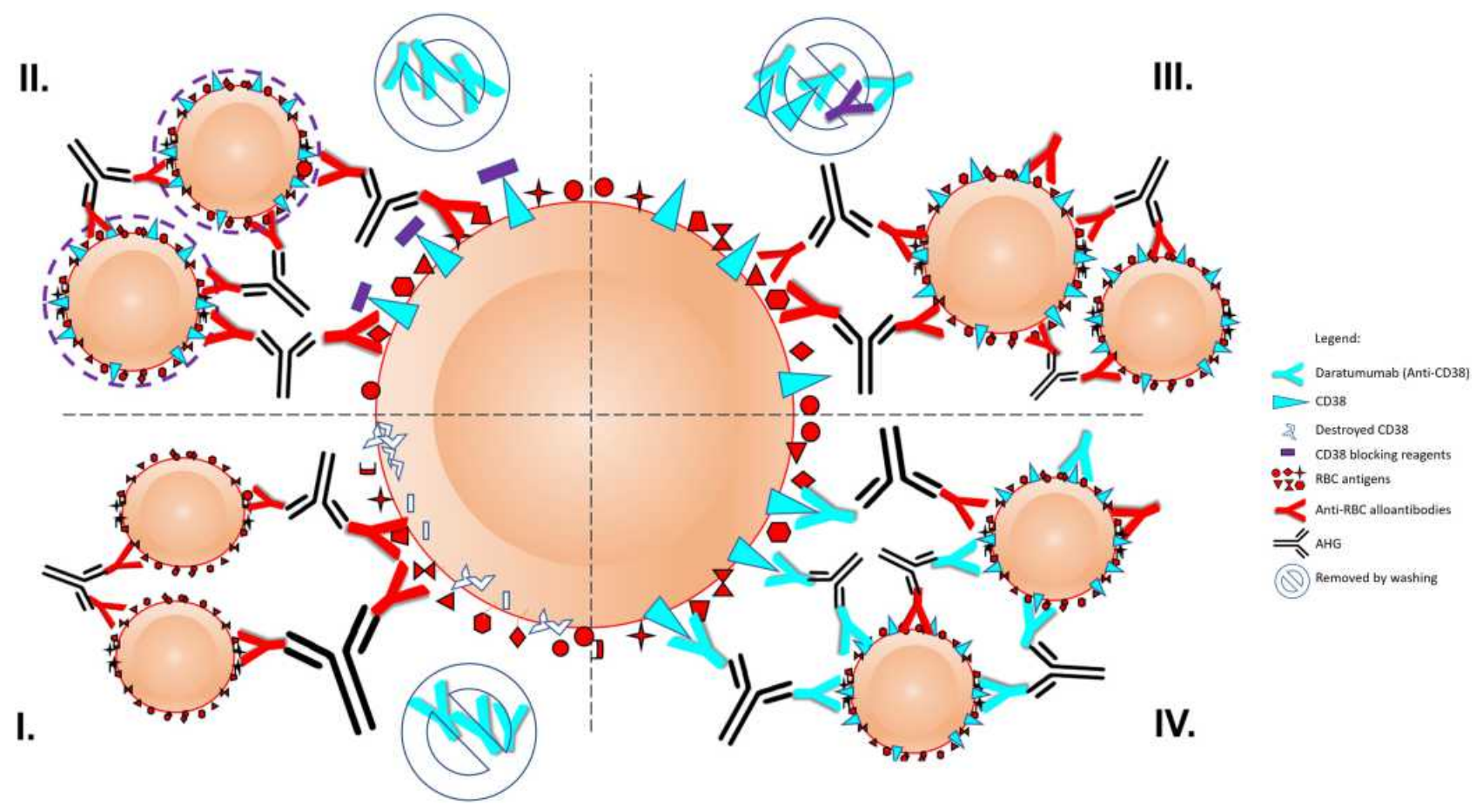

Figure I Methodologies utilized to abrogate daratumumab interference in pre-transfusion testing.

Notes: RBC agglutination due to presence of RBC specific alloantibodies or autoantibodies is not interfered by anti-CD38 after interventions I-III. I. RBCs lacking CD38 antigens used in pre-transfusion testing; II. Blocking CD38 antigens on reagent and donor cells; III. Neutralization of anti-CD38 antibody in patient plasma prior to IAT testing. IV. Extended patient antigen typing with transfusion of phenotypically matched RBC units does not require for RBC specific alloantibodies or autoantibodies to be identified (It is not necessary to distinguish if RBC agglutination is due to anti-CD38 or alloantibody or autoantibody presence).

Abbreviations: AHG, antihuman globulin; IAT, indirect antiglobulin test; RBC, red blood cells.

phosphate-buffered saline (PBS) and an automatic blood cell washing centrifuge to negate daratumumab interference while preserving $\mathrm{K}$ antigenicity. In a follow up article, the authors validated the Osaka method by using commercially available IgM anti-B (or anti-A) antibody as a quality control indicator instead of loss of Kell. ${ }^{29}$ They also investigated the ability to detect anti- $\mathrm{K}$ and anti-Ku in daratumumab treated individuals. Anti-K reactivity was detected and daratumumab interference was negated. The authors do note that the Osaka method failed to detect low-titer $(<4)$ anti-K. Large volumes of antigen typed RBCs can be treated with DTT and stored in Alsever's solution for convenience. ${ }^{30}$

Reagent red cells treated with trypsin or ficin enzyme are frequently used for ancillary testing. Trypsin does not degrade Kell antigens, but does destroy a number of other clinically significant antigens including $\mathrm{M}, \mathrm{N}$, EnaTS, Lutheran, Ge2, Ge3, Ge4 and $\mathrm{Ch} / \mathrm{Rg}$ antigens. ${ }^{26,31}$ Papain degrades antigens from Duffy and MNS blood group systems, Ge2, Ge4 and $\mathrm{Ch} / \mathrm{Rg}^{3}{ }^{32}$

There is a report that use of polybrene, a quaternary ammonium polymer, could negate the positive results for the IAT using standard PEG-antihuman globulin (PEGAHG) in patients on daratumumab therapy. ${ }^{33}$ Polybrene is used in many laboratories in Taiwan for antibody detection. The main limitation of the manual polybrene (MP) test is the lack of adequate sensitivity in the Kell system.

\section{Reagent Cells Lacking CD38 Antigen}

Schmidt et al. described testing patients' plasma against cord blood cells. ${ }^{34}$ No reactivity was observed with all cord cells. The group tested the patients' plasma against adult cells with only the "i" antigen and no "I" on the surface which showed weak to $1+$ reactivity. They concluded the cord cells express extremely low to no CD38 on their surface. They created a cord RBC panel consisting of 6-7 antigen typed cord cells and made 3\% suspensions using Alsever's solution. Tube testing with low ionic strength saline (LISS) was performed, and they report they were successful at screening and transfusing 2 daratumumab patients 17 times. The method may not be feasible in routine laboratories that do not have cord blood cells readily available. ${ }^{35}$ 


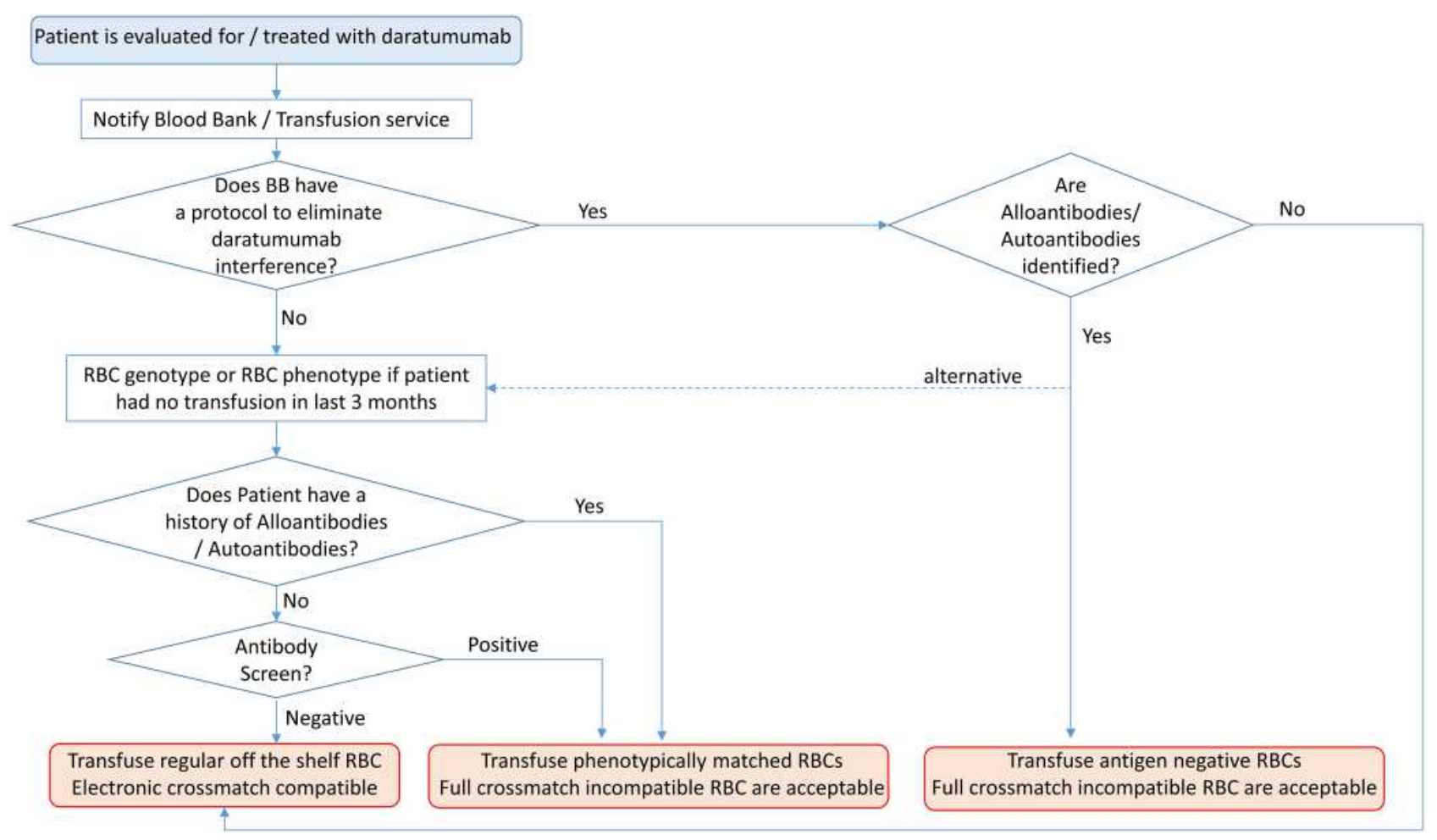

Figure 2 Algorithm for clinicians managing patients treated with daratumumab. Abbreviations: AHG, antihuman globulin; RBC, red blood cells.

Alternatively, red cells that are negative for CD38 (red cells from daratumumab patients) or In $(\mathrm{Lu})$ red cells can also be used for antibody investigations. ${ }^{36}$

\section{Blocking CD38 Antigens on Reagent or Donor Cells}

Another novel solution to daratumumab interference is pre-incubation of $\mathrm{RBC}$ with $\mathrm{F}\left(\mathrm{ab}^{\prime}\right) 2$ fragments of daratumumab. ${ }^{37} \mathrm{~F}\left(\mathrm{ab}^{\prime}\right) 2$ fragments of daratumumab are created by digestion with pepsin. $\mathrm{F}\left(\mathrm{ab}^{\prime}\right) 2$ fragments bind the same epitope on CD38 and prevent binding of RBCs by free daratumumab. Known antibodies in patients' plasma and serum (anti-D, anti-C, anti-E, anti-e, anti-K, anti-Fya, anti-Lua, anti-S, or anti-M) were detected with reproducible results. According to authors, this is a relatively easy to use method but requires commercial availability and further validations.

In another attempt to alleviate daratumumab interference but maintain the integrity of the red cell structure, Chinoca Ziza et al. proposed the blockage monoclonal antibody protocol (BMAP) ${ }^{38}$ Donor RBCs of known phenotype are treated with anti-CD38 (titer of anti-CD38 was previously determined by dilution resulting in $1+$ agglutination). These anti-CD38 treated RBCs were then blocked with monospecific anti- human $\operatorname{IgG}$ ( $\mathrm{Fc}$ specific). Their experiments showed that daratumumab interference was eliminated in 20 of 20 patients. This method would ideally be performed in parallel to DTT treatment to allow detection of alloantibodies to high prevalence antigens eliminated by DTT. The method has limitations which include technical difficulty, unavailability of anti-CD38, and need to titrate anti-CD38 prior to testing (to reduce false positives associated with heavily coated RBCs). Of note, the protocol was validated for noncommercial RBCs collected within 7 days. The method has advantages of being inexpensive, accurate, and possibly applicable in the future to help negate interference by other monoclonal drugs targeting RBC antigens. Mouse anti-CD38 neutralized the detection of human IgG observed in daratumumab treated patient serum without affecting controls. A similar approach can be explored during pre-transfusion testing. ${ }^{39}$

\section{Neutralization of Anti-CD38 Antibody in Patient Plasma Sample Prior to IAT Testing}

Oostendorp et al. successfully blocked interference by daratumumab and other similar anti-CD38 monoclonal antibodies using an anti-idiotype antibody and recombinant soluble CD38 
extracellular domain protein (sCD38). ${ }^{20}$ These two solutions permitted correct irregular antibody identification. Work by Oostendorp et al. is consistent with the work of Chapuy et al. documenting inhibited binding of daratumumab to CD38 +HL60 cells with the addition of SCD38 which reduced daratumumab binding in a dose dependent manner. ${ }^{25}$ Neutralization with anti-idiotype antibody reduced binding by $95 \%$. The neutralization methods are highly effective and simpler to perform than DTT treatment of RBCs. Although costs are higher and large amounts of SCD38 would be required to treat samples from patients on daratumumab, the primary obstacle is limited availability of reagents. In contrast, DTT is relatively inexpensive and already standardized by blood banks.

Another method for overcoming daratumumab interference is the use of Daudi cell stroma generated by sonication. ${ }^{40}$ Daudi B-cell stroma expresses high levels of CD38. Adsorbing a sample of plasma with Daudi cell stroma led to removal of daratumumab interference while allowing detection of alloantibodies such as anti-K, antiYta, and anti-Gya. According to authors, Daudi cell stroma is relatively inexpensive and large quantities can be stored and frozen for convenience. Interestingly, Tremblay et al. compared the AABB standard 0.2 M DTT method with the Osaka method, and their own Daudi cell stroma method. Treatment with Daudi cell stroma did not affect anti-Jsb detection and displayed reduced reactivity of anti-K. Although testing with the Osaka method reduced daratumumab interference, there was still $\mathrm{K}$ antigen loss, denaturing of Jsb, and less strong reaction with anti-K than without $0.01 \mathrm{M}$ DTT treatment. Their findings suggest adsorption with Daudi cell stroma cells removes anti-CD38 without compromising antibody detection and identification. The main limitation is the standardization of the method and the requirement of specialized equipment such as an ultrasonic processor and centrifuge with performance up to $20,000 \mathrm{~g}$.

A high affinity single-stranded DNA oligonucleotides, aptamers, specific to daratumumab can also be used to neutralize binding to $\mathrm{CD} 38 .^{41}$

\section{Establishing Baseline Phenotype or Genotype Prior to Starting Treatment with Daratumumab}

Additional blood bank methods include RBC phenotyping and genotyping. Antigen phenotyping is a routine serologic procedure and aids in selection of phenotype similar
RBC units for transfusion. ${ }^{35,42}$ Extended RBC phenotyping should be performed prior to treatment with daratumumab in patients who have not been transfused in the prior 3 months.

Genotyping can be performed after initiation of antiCD38 treatment but is expensive and can be associated with increased turnaround times for results. ${ }^{36}$ One of the benefits to always providing phenotypically similar RBCs is the reduced risk of sensitization and future alloantibody formation for the red cell antigens that are matched. AntiRBC alloantibodies that may be present will not react against matched RBC units. AHG crossmatches with phenotypically or genotypically matched units will still be crossmatch incompatible due to the presence of AntiCD38 in the patient sample.

\section{Transfusion of RBC Units with Incompatible Crossmatches}

To aid transfusion services, the AABB published a bulletin with recommendations for handling daratumumab interference. ${ }^{43}$ Prior to daratumumab therapy, a baseline type and screen should be performed; a baseline genotype or phenotype is also recommended. After therapy, antibody screening and identification should be performed using DTT treated cells, and K-negative units should be issued unless the patient is known to be K-positive. Crossmatch should be performed as per standard requirements. Alternatively, an AHG crossmatch may be performed using DTT-treated donor cells. For patients with no history of antibodies and a negative antibody screen using DTT-treated cells, an electronic or immediate-spin crossmatch with $\mathrm{ABO} / \mathrm{RhD}$-compatible, K-matched units may be performed. If an emergency transfusion is required, uncrossmatched $\mathrm{ABO} / \mathrm{RhD}$-compatible $\mathrm{RBCs}$ may be given per local blood bank practices. Current evidence shows no increased risk of hemolysis after transfusion in patients treated with daratumumab. ${ }^{44}$ Further, a recent article by $\mathrm{Ye}$ et al. found no significant daratumumab associated difference in alloimmunization risk between $45 \mathrm{MM}$ patients receiving transfusions on daratumumab and $46 \mathrm{MM}$ patient receiving transfusions but not on daratumumab as the control group. The study is limited due to the number of patients enrolled. It may be useful to study larger cohorts of patients receiving daratumumab to investigate if there is a clinically significant increase in risk of alloimmunization. ${ }^{45}$ 


\section{Impact of Daratumumab on Transfusion Service Costs}

Anani et al retrospectively reviewed 62 patients on daratumumab and compared the costs of: DTT method with K-negative $\mathrm{RBC}$ transfusion, patient phenotyping or genotyping with antigen matched transfusion, and combination of genotyping and DTT treated RBC investigation with genotype predicted antigen-matched RBC transfusion. ${ }^{46}$ They found that genotyping was most cost effective in patients requiring long-term transfusion when matching less than 4 antigens. Cushing et al. published a detailed cost analysis comparing theoretical universal genotyping with a provision of phenotypically similar RBC transfusions versus implementation of a standardized notification and testing/transfusion algorithm, an approach combining DTT-based testing with selective genotyping and the provision of phenotypically similar RBCs only for patients with clinically significant antibodies. ${ }^{47}$ The targeted, standardized approach was found to be more cost effective in a cohort of patients followed over a year. Cost-related considerations are especially important for mitigating daratumumab interference in national general blood bank services and for blood banks in developing countries where specialized reagent are not readily available. $^{48}$

\section{Conclusion}

The prevalence of monoclonal antibody therapies will only continue to rise. Daratumumab epitomizes the need for the recognition of potential interferences of therapeutic agents with pre-transfusion testing, the development of appropriate methodologies to overcome these interferences as well as good communication between the blood bank and the treating physician in order to secure safe blood products for the patient. Established blood banking pre-transfusion testing standards and practices for the clinical management of patients undergoing blood transfusions serve as building blocks for creating pre-transfusion testing protocols for those patients receiving monoclonal antibody therapies. As the clinical use of daratumumab increased, several methodologies have been utilized to resolve daratumumab interference during pre-transfusion testing, which have been discussed in this article. Equally as important is the timely communication between the transfusion service and clinical teams. Clinical providers should be aware of daratumumab interference and should contact the blood bank prior to start of Daratumumab therapy to ensure the patient has a baseline type and screen, and that blood bank has a protocol to negate Daratumumab interference. Emphasis on educational efforts by blood banking and clinical professional societies are also key for continued blood transfusion safety.

\section{Abbreviations}

DTT, dithiothreitol; AHG, antihuman globulin; IAT, indirect antiglobulin test; MM, multiple myeloma; RBC, red blood cells.

\section{Disclosure}

The authors report no conflicts of interest in this work.

\section{References}

1. Fung MG, Hillyer BJ. AABB Technical Manual. 18th ed. AABB; 2014.

2. Pietsch EC, Dong J, Cardoso R, et al. Anti-leukemic activity and tolerability of anti-human CD47 monoclonal antibodies. Blood Cancer J. 2017;7(2):e536. doi:10.1038/bcj.2017.7

3. Tong B, Wang M. CD47 is a novel potent immunotherapy target in human malignancies: current studies and future promises. Future Oncol. 2018;14(21):2179-2188. doi:10.2217/fon-2018-0035

4. Palumbo A, Chanan-Khan A, Weisel K, et al. Daratumumab, bortezomib, and dexamethasone for multiple myeloma. $N$ Engl J Med. 2016;375(8):754-766. doi:10.1056/NEJMoa1606038

5. Kaufman GP, Schrier SL, Lafayette RA, Arai S, Witteles RM, Liedtke M. Daratumumab yields rapid and deep hematologic responses in patients with heavily pretreated $\mathrm{AL}$ amyloidosis. Blood. 2017;130(7):900-902. doi:10.1182/blood-2017-01-763599

6. Cancer Facts \& Figures 2021. American Cancer Society journal, CA: a cancer journal for clinicians. Available from: https://www.cancer. org/research/cancer-facts-statistics/all-cancer-facts-figures/cancerfacts-figures-2021.html. Accessed May 3, 2021.

7. Plesner T, Arkenau HT, Gimsing P, et al. Phase $1 / 2$ study of daratumumab, lenalidomide, and dexamethasone for relapsed multiple myeloma. Blood. 2016;128(14):1821-1828. doi:10.1182/blood2016-07-726729

8. Albeniz I, Demir Ö, Turker-Sener L, Yalcintepe L, Nurten R, Bermek E. Erythrocyte CD38 as a prognostic marker in cancer. Hematology. 2007;12(5):409-414. doi:10.1080/10245330701383841

9. de Weers M, Tai YT, van der Veer MS, et al. Daratumumab, a novel therapeutic human CD38 monoclonal antibody, induces killing of multiple myeloma and other hematological tumors. J Immunol. 2011;186(3):1840-1848. doi:10.4049/jimmunol.1003032

10. McKeage K. Daratumumab: first global approval. Drugs. 2016;76 (2):275-281. doi:10.1007/s40265-015-0536-1

11. Clark CA, Cornell RF, Scott EC, Chung J, Costa LJ. Management of relapsed and refractory multiple myeloma in modern times: incorporating new agents into decision-making. Am J Hematol. 2016;91 (10):1044-1051. doi:10.1002/ajh.24478

12. Darzalex package insert. Horsham, PA: Janssen Biotech; 2015. Available from: http://wwwdarzalexcom/shared/product/darzalex/dar zalex-prescribing-informationpdf. Accessed March 3, 2021.

13. Chapuy CI, Kaufman RM, Alyea EP, Connors JM. Daratumumab for delayed red-cell engraftment after allogeneic transplantation. $N$ Engl J Med. 2018;379(19):1846-1850. doi:10.1056/NEJMoa1807438

14. Mirgh S, Ahmed R, Agrawal N, et al. Will daratumumab be the next game changer in early thymic precursor-acute lymphoblastic leukaemia? $B r J$ Haematol. 2019;187(2):e33-e35. doi:10.1111/ bjh. 16154 
15. van de Donk N, Richardson PG, Malavasi F. CD38 antibodies in multiple myeloma: back to the future. Blood. 2017;131(1):13-29. doi:10.1182/blood-2017-06-740944

16. Sullivan HC, Gerner-Smidt C, Nooka AK, et al. Daratumumab (anti-CD38) induces loss of CD38 on red blood cells. Blood. 2017;129(22):3033-3037. doi:10.1182/blood-2016-11-749432

17. Chari A, Arinsburg S, Jagannath S, et al. Blood transfusion management and transfusion-related outcomes in daratumumab-treated patients with relapsed or refractory multiple myeloma. Clin Lymphoma Myeloma Leuk; 2017.

18. Schuetz C, Hoenig M, Moshous D, et al. Daratumumab in life-threatening autoimmune hemolytic anemia following hematopoietic stem cell transplantation. Blood Adv. 2018;2(19):2550-2553. doi:10.1182/bloodadvances. 2018020883

19. Murphy MF, Dumont LJ, Greinacher A. Interference of new drugs with compatibility testing for blood transfusion. $N$ Engl $\mathrm{J} \mathrm{Med}$. 2016;375(3):295-296. doi:10.1056/NEJMc1515969

20. Oostendorp M, Lammerts van Bueren JJ, Doshi P, et al. When blood transfusion medicine becomes complicated due to interference by monoclonal antibody therapy. Transfusion. 2015;55(6 Pt 2):1555-1562. doi:10.1111/trf.13150

21. Vidal-Crespo A, Matas-Céspedes A, Rodriguez V, et al. Daratumumab displays in vitro and in vivo anti-tumor activity in models of B-cell non-Hodgkin lymphoma and improves responses to standard chemo-immunotherapy regimens. Haematologica. 2020;105(4):1032-1041. doi:10.3324/haematol.2018.211904

22. Salles G, Gopal AK, Minnema MC, et al. Phase 2 study of daratumumab in relapsed/refractory mantle-cell lymphoma, diffuse large B-cell lymphoma, and follicular lymphoma. Clin Lymphoma Myeloma Leuk. 2019;19(5):275-284. doi:10.1016/j.clml.2018.12.013

23. Berthelier V, Laboureau J, Boulla G, Schuber F, Deterre P. Probing ligand-induced conformational changes of human CD38. Eur J Biochem. 2000;267(10):3056-3064. doi:10.1046/j.14321033.2000.01329.x

24. Anani WQ, Duffer K, Kaufman RM, Denomme GA. How do I work up pretransfusion samples containing anti-CD38? Transfusion. 2017;57(6):1337-1342. doi:10.1111/trf.14144

25. Chapuy CI, Nicholson RT, Aguad MD, et al. Resolving the daratumumab interference with blood compatibility testing. Transfusion. 2015;55(6 Pt 2):1545-1554. doi:10.1111/trf.13069

26. M L-fc R, Olsson ML. The Blood Group Antigen Factsbook. 3rd ed. MA: Academic Press; 2012.

27. Chapuy CI, Aguad MD, Nicholson RT, et al. International validation of a dithiothreitol (DTT)-based method to resolve the daratumumab interference with blood compatibility testing. Transfusion. 2016;56 (12):2964-2972. doi:10.1111/trf.13789

28. Hosokawa M, Kashiwagi H, Nakayama K, et al. Distinct effects of daratumumab on indirect and direct antiglobulin tests: a new method employing $0.01 \mathrm{~mol} / \mathrm{L}$ dithiothreitol for negating the daratumumab interference with preserving $\mathrm{K}$ antigenicity (Osaka method). Transfusion. 2018;58(12):3003-3013. doi:10.1111/trf.14900

29. Hosokawa M, Kashiwagi H, Nakayama K, et al. Additional validation of Osaka method $(0.01 \mathrm{~mol} / \mathrm{L}$ dithiothreitol $)$ for negating the daratumumab interference. Transfusion. 2019;59(7):2479-2480. doi:10.1111/trf.15305

30. Disbro WL. Stability guidelines for dithiothreitol-treated red blood cell reagents used for antibody detection methods in patients treated with daratumumab. Immunohematology. 2017;33(3):105-109. doi:10.21307/immunohematology-2019-015

31. Lancman G, Arinsburg S, Jhang J, et al. Blood transfusion management for patients treated with anti-cd38 monoclonal antibodies. Front Immunol. 2018;9:2616. doi:10.3389/fimmu.2018.02616
32. Bub CB, Reis IND, Aravechia MG, et al. Transfusion management for patients taking an anti-CD38 monoclonal antibody. Rev Bras Hematol Hemoter. 2018;40(1):25-29. doi:10.1016/j.bjhh.2017.09.003

33. Yeh TJ, Yeh CJ, Liu YC, Hsiao HH. Manual polybrene method for pretransfusion test could overcome the interference of daratumumab therapy in myeloma. Transfusion. 2019;59(8):2751-2752. doi:10.1111/trf.15341

34. Schmidt AE, Kirkley S, Patel N, et al. An alternative method to dithiothreitol treatment for antibody screening in patients receiving daratumumab. Transfusion. 2015;55(9):2292-2293. doi:10.1111/ trf. 13174

35. De Vooght KM, Oostendorp M, van Solinge WW. Dealing with anti-CD38 (daratumumab) interference in blood compatibility testing. Transfusion. 2016;56(3):778-779. doi:10.1111/trf.13474

36. Quach H, Benson S, Haysom H, et al. Considerations for pre-transfusion immunohaematology testing in patients receiving the anti-CD38 monoclonal antibody daratumumab for the treatment of multiple myeloma. Intern Med J. 2018;48(2):210-220. doi:10.1111/imj.13707

37. Selleng K, Gebicka PD, Thiele TF. (ab')2 fragments to overcome daratumumab interference in transfusion tests. $N$ Engl J Med. 2018;379(1):90-91. doi:10.1056/NEJMc1804751

38. Chinoca Ziza KN, Paiva TA, Mota SR, et al. A blockage monoclonal antibody protocol as an alternative strategy to avoid anti-CD38 interference in immunohematological testing. Transfusion. 2019;59 (5):1827-1835. doi:10.1111/trf.15202

39. Baig NA, Dukek BA, Falbo DK, et al. Daratumumab interference in flow cytometric anti-granulocyte antibody testing can be overcome using non-human blocking antibodies. Vox Sang. 2021;116 (1):116-122. doi:10.1111/vox.12989

40. Tremblay T, Branch DR, Loubaki L. Daudi cell stroma: an alternative to dithiothreitol to resolve daratumumab interference in pretransfusion testing. Transfusion. 2020;60(9):2090-2096. doi:10.1111/ trf. 15932

41. Tapia-Alveal C, Olsen TR, Worgall TS. Personalized immunoglobulin aptamers for detection of multiple myeloma minimal residual disease in serum. Commun Biol. 2020;3(1):781. doi:10.1038/ s42003-020-01515-x

42. Hannon JL, Clarke G. Transfusion management of patients receiving daratumumab therapy for advanced plasma cell myeloma. Transfusion. 2015;55(11):2770. doi:10.1111/trf.13267

43. Mitigating the anti-CD38 interference with serologic testing AABB association bulletin \#16-02; 2016. Available from: http:/www.aabb. org/programs/publications/bulletins/Documents/ab16-02.pdf. Accessed May 3, 2021.

44. Chari A, Arinsburg S, Jagannath S, et al. Blood transfusion management and transfusion-related outcomes in daratumumab-treated patients with relapsed or refractory multiple myeloma. Clin Lymphoma Myeloma Leuk. 2018;18(1):44-51. doi:10.1016/j. clml.2017.09.002

45. Ye Z, Wolf LA, Mettman D, Plapp FV. Risk of RBC alloimmunization in multiple myeloma patients treated by daratumumab. Vox Sang. 2020;115(2):207-212. doi:10.1111/vox.12864

46. Anani WQ, Marchan MG, Bensing KM, et al. Practical approaches and costs for provisioning safe transfusions during anti-CD38 therapy. Transfusion. 2017;57(6):1470-1479. doi:10.1111/trf.14021

47. Cushing MM, DeSimone RA, Goel R, et al. The impact of daratumumab on transfusion service costs. Transfusion. 2019;59 (4):1252-1258. doi:10.1111/trf.15134

48. Setia R, Dogra M, Sachdeva P, Handoo A, Choudhary D, Agarwal A Daratumumab (Anti-CD38) interference with serological testing: an emerging challenge for blood banks in developing countries. Glob J Transfus Med. 2017;2(2):163. doi:10.4103/GJTM.GJTM_42_17 


\section{Publish your work in this journal}

The Journal of Blood Medicine is an international, peer-reviewed, open access, online journal publishing laboratory, experimental and clinical aspects of all aspect pertaining to blood based medicine including but not limited to: Transfusion Medicine; Blood collection, Donor issues, Transmittable diseases, and Blood banking logistics; Immunohematology; Artificial and alternative blood based therapeutics; Hematology; Biotechnology/nanotechnology of blood related medicine; Legal aspects of blood medicine; Historical perspectives. The manuscript management system is completely online and includes a very quick and fair peer-review system. Visit http://www.dovepress.com/testimonials.php to read real quotes from published authors. 